\title{
Effect of Treatment on Damage and Hospitalization in Elderly Patients with Microscopic Polyangiitis and Granulomatosis with Polyangiitis
}

\author{
Maria Weiner, Su Mein Goh, Aladdin J. Mohammad (D), Zdenka Hrušková (iD, Anisha Tanna, \\ Phoebe Sharp, Amy Kang, Annette Bruchfeld (iD, Daina Selga, Zdeňka Chocová, \\ Kerstin Westman (D), Per Eriksson, Lorraine Harper, Charles D. Pusey, Vladimír Tesař (iD, \\ Alan D. Salama (1), and Mårten Segelmark (i)
}

ABSTRACT. Objective. Age is a risk factor for organ damage, adverse events, and mortality in microscopic polyangiitis (MPA) and granulomatosis with polyangiitis (GPA). However, the relationship between treatment and damage, hospitalizations, and causes of death in elderly patients is largely unknown. Methods. Consecutive patients from Sweden, the United Kingdom, and the Czech Republic diagnosed between 1997 and 2013 were included. Inclusion criteria were a diagnosis of MPA or GPA and age 75 years or more at diagnosis. Treatment with cyclophosphamide (CYC), rituximab (RTX), and corticosteroids the first 3 months was registered. Outcomes up to 2 years from diagnosis included Vasculitis Damage Index (VDI), hospitalization, and cause of death.

Results. Treatment data were available for 167 of 202 patients. At 2 years, $4 \%$ had no items of damage. There was a positive association between VDI score at 2 years and Birmingham Vasculitis Activity Score at onset, and a negative association with treatment using CYC or RTX. Intravenous methylprednisolone dose was associated with treatment-related damage. During the first year, $69 \%$ of patients were readmitted to hospital. Myeloperoxidase-antineutrophil cytoplasmic antibody positivity and lower creatinine levels decreased the odds of readmission. The most common cause of death was infection, and this was associated with cumulative oral prednisolone dose.

Conclusion. Immunosuppressive treatment with CYC or RTX in elderly patients with MPA and GPA was associated with development of less permanent organ damage and was not associated with hospitalization. However, higher doses of corticosteroids during the first 3 months was associated with treatment-related damage and fatal infections. (First Release December 15 2019; J Rheumatol 2020; 47:580-8; doi:10.3899/jrheum.190019)

Key Indexing Terms:

ANTINEUTROPHIL CYTOPLASMIC ANTIBODY-ASSOCIATED VASCULITIS

GRANULOMATOSIS WITH POLYANGIITIS MICROSCOPIC POLYANGIITIS

HOSPITALIZATION

\begin{abstract}
From the Department of Nephrology and Department of Medical and Health Sciences, Department of Rheumatology and Department of Clinical and Experimental Medicine, Linköping University, Linköping; Department of Clinical Sciences, Section of Rheumatology, Lund University, Lund; Department of Renal Medicine, Karolinska University Hospital and CLINTEC Karolinska Institute, Stockholm; Department of Nephrology, Lund University, Skåne University Hospital, Lund and Malmö; University College London Centre for Nephrology, Royal Free Hospital; Department of Medicine, Imperial College London, London; Institute of Clinical Sciences, University of Birmingham, Birmingham; Department of Medicine, University of Cambridge, Cambridge, UK, Department of Nephrology, First Faculty of Medicine, Charles University and General University Hospital, Prague, Czech Republic; Department of Renal Medicine, Tan Tock Seng Hospital, Singapore.

This work was supported by grants from the Ingrid Asp Foundation, the Swedish Renal Foundation, and the National Institute for Health Research Imperial Biomedical Research Centre.

M. Weiner, MD, Department of Nephrology and Department of Medical and Health Sciences, Linköping University; S.M. Goh, MBBS, University College London Centre for Nephrology, Royal Free Hospital, and Department of Renal Medicine, Tan Tock Seng Hospital; A.J. Mohammad, MD, PhD, Department of Clinical Sciences, Section of Rheumatology, Lund University, and Department of Medicine, University of Cambridge; Z. Hrušková, MD, PhD, Department of Nephrology, First Faculty of
\end{abstract}


Microscopic polyangiitis (MPA) and granulomatosis with polyangiitis (GPA; formerly Wegener granulomatosis) are part of the disease spectrum called antineutrophil cytoplasmic antibody (ANCA)-associated vasculitis (AAV), which also includes the clinically different and less common eosinophilic granulomatosis with polyangiitis (EGPA; formerly Churg-Strauss syndrome $)^{1,2}$. Patients with AAV are often of older age; peak incidence is found in patients aged above $65-75$ years ${ }^{3,4}$.

Treating patients with vasculitis is a delicate balance between the need for immunosuppression to attenuate the vasculitic activity and the risk of adverse effects of treatment ${ }^{5,6}$. In the general AAV population, therapy-related adverse events are more commonly the cause of death than active vasculitis. Because the risk of adverse events increases with age and decreased renal function, elderly patients can be expected to be at high risk for both treatment-related damage and mortality ${ }^{7}$.

Morbidity in AAV is assessed using the Vasculitis Damage Index (VDI), a clinical tool recording all permanent damage occurring after the onset of vasculitis. The damage items are scored regardless of their attribution and can thus reflect both the accumulated longterm effects of the vasculitic disease and of therapy ${ }^{8}$. Over time, most patients with vasculitis develop some degree of permanent organ damage ${ }^{9}$. Previous studies have shown an association between higher age, lower estimated glomerular filtration rate (eGFR), and the total number of damage items at followup ${ }^{10}$. Both total damage and treatment-related damage are more common in older patients ${ }^{11}$. There is also an association between use of glucocorticoids (GC) and development of permanent organ damage ${ }^{10,12}$.

We have previously reported that elderly patients with MPA and GPA who had received immunosuppressive treatment with cyclophosphamide (CYC) or rituximab (RTX) had significantly better survival compared to patients who had not been given such treatment ${ }^{13}$. However, it is not known whether this survival benefit is at the expense of increased morbidity and the need for longer and more frequent hospitalizations. Neither is it known whether the mortality pattern seen in younger patients with AAV also applies to elderly patients. Older patients in general use more healthcare resources than younger patients ${ }^{14}$, but the need for in-hospital care during the early period after diagnosis has not previously been described for elderly patients with MPA and GPA.

The aim of our study was to investigate the presence of permanent organ damage, hospitalization patterns, and causes of death, and to assess the potential association with the initial treatment regimens, in patients aged 75 years or more with MPA or GPA.

\section{MATERIALS AND METHODS}

Case retrieval and classification. Consecutive patients presenting at 7 centers in Sweden, the United Kingdom, and the Czech Republic between 1997 and 2013 were included if they were aged 75 years or more at diagnosis and had a clinical diagnosis of MPA or GPA according to the European Medicines Agency algorithm ${ }^{15}$. Exclusion criteria were EGPA, polyarteritis nodosa, secondary vasculitis, drug-induced vasculitis, and antiglomerular basement membrane disease ${ }^{15}$.

The Swedish cohort was recruited from Linköping University Hospital, Skåne University Hospital in Lund and Malmö, and Karolinska University Hospital in Stockholm. The English cohort was recruited from Imperial College Renal and Transplant Centre and Royal Free Hospital in London, and Queen Elizabeth Hospital in Birmingham. The Czech cohort was recruited from General University Hospital in Prague. The participating centers have been described in detail previously ${ }^{13}$. The study was approved by the Ethical Review Board in Lund, Sweden (registration numbers 2010/517; 2012/252).

Data collection. Data were collected retrospectively from databases and medical records. End of followup was at 2 years from diagnosis, death, or loss to followup. Data collected from time of diagnosis included diagnosis date, age, sex, disease phenotype (MPA/GPA), ANCA specificity, C-reactive protein (CRP), creatinine (at diagnosis or before start of dialysis in patients dialysis-dependent at diagnosis), disease activity according to Birmingham Vasculitis Activity Score (BVAS) ${ }^{16}$, and major comorbidities.

Data on outcome included accumulated organ damage according to VDI in patients alive at 1 and 2 years, hospitalization (for any reason) during the first year, and causes of death during the first 2 years. Treatment with RTX and cumulative doses of CYC and GC [pulsed intravenous (IV) methylprednisolone and oral prednisolone (PSL)] during the first 3 months after diagnosis was recorded.

Date of diagnosis was defined as follows: start of treatment with $\mathrm{PSL} \geq 30 \mathrm{mg} /$ day, plasma exchange or CYC; if not treated, the day of biopsy; if no biopsy, the day of the first positive ANCA test. GFR was estimated using the Modification of Diet in Renal Disease equation ${ }^{17}$. In assessing VDI, an eGFR of $\leq 50 \mathrm{ml} / \mathrm{min} / 1.73 \mathrm{~m}^{2}$ was considered $\mathrm{GFR} \leq 50 \%$ irrespective of age, and a dipstick value of $\geq 2$ was considered representative of 24-h proteinuria of $\geq 0.5 \mathrm{~g}$. A dipstick value of $\geq 2$ was considered representative of hematuria equal to 10 red blood cells per high power field when assessing BVAS. Indirect immunofluorescence or antigen-specific ELISA was used to detect ANCA. Treatment-related VDI items were defined as described by Exley, et al ${ }^{18}$ and included osteoporosis, avascular necrosis, osteomyelitis, cataract, gonadal failure, marrow failure, chemical cystitis, diabetes mellitus, and malignancy. A comorbidity score was assessed using a modified version of the Davies score ${ }^{19}$, with 1 point each given for malignancy, ischemic heart disease, peripheral vascular disease, heart failure, diabetes, systemic inflammatory disease (excluding AAV), pulmonary disease, and cirrhosis. Relapses were defined as an increase in BVAS to $\geq 1$ and increased immunosuppressive therapy.

Statistical analyses. Statistical analysis was performed using SPSS Statistics for Windows software (version 24.0; IBM Corp.). P values $<0.05$ were considered significant. Differences between groups were analyzed using the Mann-Whitney $U$ test or Kruskal-Wallis test for nonparametric data. Categorical data were analyzed using the chi-square test or Fisher's exact test. All analyses exclude missing data.

In analysis of outcome, patients who died within the first 30 days after diagnosis and patients without complete treatment data were excluded. Patients were divided into 3 treatment groups: RTX group (any dose of RTX), CYC group ( $\geq 2000 \mathrm{mg}$ oral $/ \geq 1500 \mathrm{mg}$ IV CYC during first $3 \mathrm{mos}$ ), and No/other group $[<2000 \mathrm{mg}$ oral $/<1500 \mathrm{mg}$ IV CYC, azathioprine, mycophenolate mofetil (MMF), methotrexate (MTX), steroids only, no treatment].

Binary logistic regression analysis was used to analyze hospital readmission and treatment-related damage (binary variables), linear regression analysis to analyze total hospital stay and VDI score (continuous variables), and Cox regression analysis to analyze mortality (time-dependent variable). The variables used in multivariable analyses were chosen to reflect patient characteristics (age, sex, and comorbidity score), disease severity (BVAS, CRP, and creatinine), disease type (ANCA serotype), and treatment (CYC/RTX, oral PSL dose, and pulsed IV methylprednisolone dose). In analysis of VDI at 2 years, year of diagnosis as a continuous variable (from

Personal non-commercial use only. The Journal of Rheumatology Copyright (c) 2020. All rights reserved. 
1997 onward) was also included. In analyses using ANCA serotype as a binary variable, double-positive patients were designated as having either myeloperoxidase-ANCA (MPO-ANCA)/perinuclear ANCA (pANCA) or proteinase 3-ANCA (PR3-ANCA)/cytoplasmic ANCA (cANCA), depending on the highest titer.

\section{RESULTS}

Baseline patient characteristics. A total of 202 patients were included. For the outcome analyses, only patients alive after 30 days with complete treatment data were included $(\mathrm{n}=167)$. The flow of patients is shown in Figure 1 and detailed patient characteristics in Table 1. Median age at diagnosis was 79 years [interquartile range (IQR) 77-82]. MPA was diagnosed in $69.8 \%$ and GPA in $30.2 \%$ of patients. $\mathrm{MPO} / \mathrm{pANCA}$ was seen in $62 \%$, PR3/cANCA in $33.5 \%$, double positivity in $1 \%$, and ANCA negativity in $3.5 \%$. Renal involvement was seen in $90.7 \%$ of the patients (Sweden 89.2\%, UK 93.9\%, and Czech Republic 92.1\%; $\mathrm{p}=0.67$ ). Median creatinine at diagnosis was $278 \mu \mathrm{mol} / \mathrm{l}$ (IQR 141-439). In MPO/pANCA-positive patients it was 315 (IQR 171-491) and in PR3/cANCA-positive patients, 217 (IQR 95-386; $\mathrm{p}=0.04$ ). Median BVAS was 15 (IQR 12-19).
Treatment. Patients were divided into 3 different treatment groups: CYC $(n=112)$, RTX $(n=24)$, and No/other $(n=31)$. Methylprednisolone was given in $45.5 \%$ of the patients during the first 3 months after diagnosis $(48.2 \%$ in CYC group, $62.5 \%$ in RTX group, and $22.6 \%$ in No/other group). Median cumulative doses of oral PSL, CYC, and IV methylprednisolone during the first 3 months are shown in Table 2. In the No/other group, 7 patients received no treatment, 3 patients steroids only, 9 patients low doses of CYC, 8 patients azathioprine, 3 patients MTX, and 1 patient MMF.

VDI. Median VDI score was 2 (IQR 1-3) both at 1 and 2 years. At 1 year, $5.0 \%(6 / 121)$ of the patients had no items of damage and $5.0 \%$ had 5 or more items. The corresponding figures at 2 years were $3.7 \%(4 / 108)$ and $6.5 \%$ (7/108), respectively. Damage in the renal domain was most common, followed by cardiovascular and neuropsychiatric damage (Figure 2A). Endstage renal disease constituted 20.5\% $(18 / 88)$ and $18.3 \%(15 / 82)$ of the damage in the renal domain at 1 and 2 years, respectively.

Treatment-related VDI items were present in $21.4 \%$ $(25 / 117)$ of the patients at 1 year and in $26.9 \%(28 / 104)$ at 2

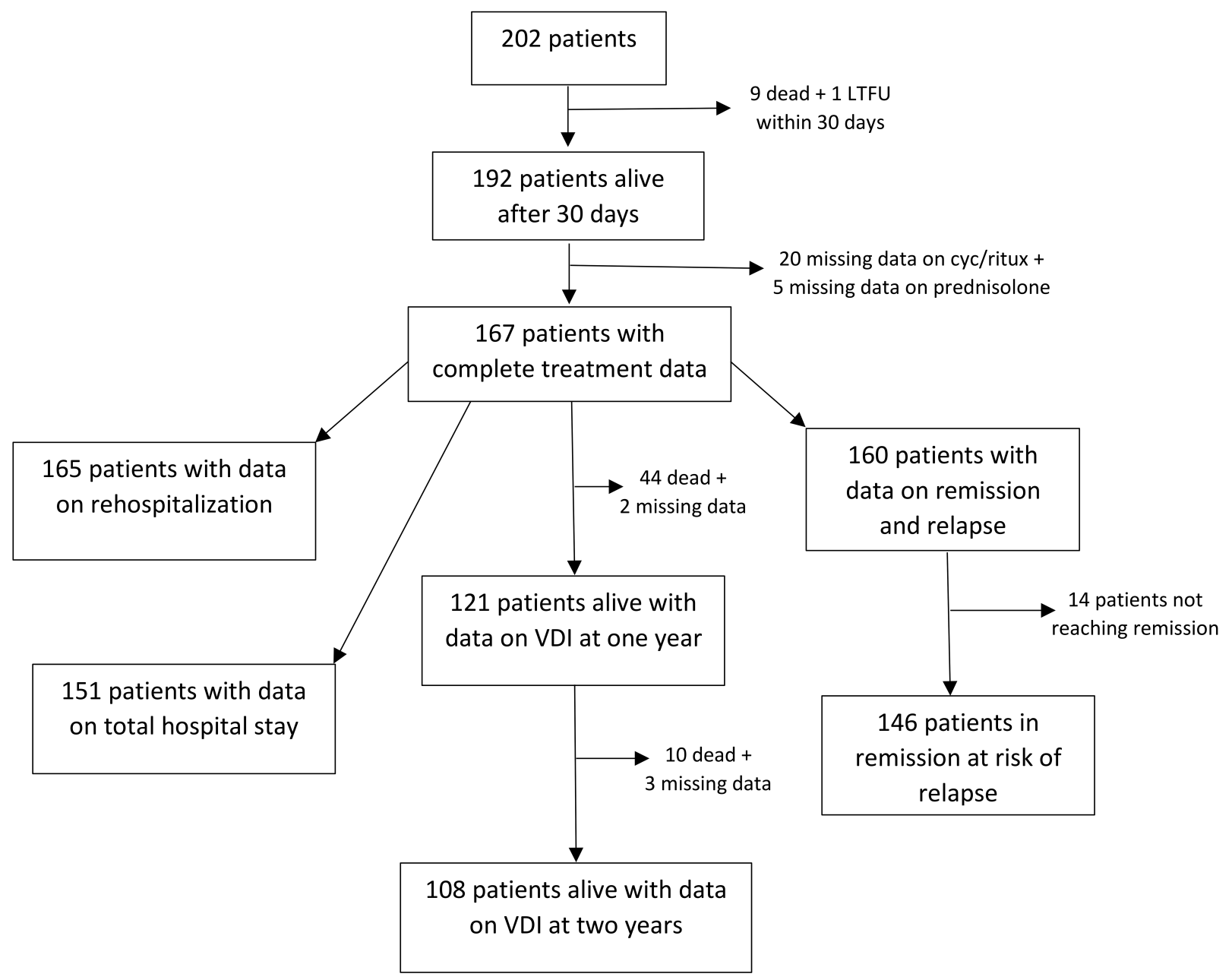

Figure 1. Flow of patients in the study. CYC: cyclophosphamide; ritux: rituximab; LTFU: lost to followup; VDI: Vasculitis Damage Index. 
Table 1. Demographic and clinical factors at time of diagnosis.

\begin{tabular}{|c|c|c|c|c|}
\hline \multirow[t]{2}{*}{ Variables } & \multicolumn{4}{|c|}{$\begin{array}{c}\text { Patients Alive after } 30 \text { Days and with } \\
\text { Complete Treatment Data }\end{array}$} \\
\hline & $\begin{array}{l}\text { All Patients, } \\
\mathrm{n}=202\end{array}$ & Yes, $\mathrm{n}=167$ & No, $n=35$ & $\mathrm{p}$ \\
\hline \multicolumn{5}{|l|}{ Sex } \\
\hline Female & $108(53.5)$ & $91(54.5)$ & $17(48.6)$ & 0.52 \\
\hline Male & $94(46.5)$ & $76(45.5)$ & $18(51.4)$ & \\
\hline \multicolumn{5}{|l|}{ Diagnosis } \\
\hline MPA & $141(69.8)$ & $117(70.1)$ & $24(68.6)$ & 0.86 \\
\hline GPA & $61(30.2)$ & $50(29.9)$ & $11(31.4)$ & \\
\hline Median age, yrs & $79(77-82)$ & $79(77-82)$ & $80(77-85)$ & 0.25 \\
\hline \multicolumn{5}{|l|}{$\mathrm{ANCA}^{1}$} \\
\hline MPO/pANCA & $124(62.0)$ & $107(64.5)$ & $17(50.0)$ & $<0.001$ \\
\hline PR3/cANCA & $67(33.5)$ & $56(33.7)$ & $11(32.4)$ & \\
\hline Double-positive & $2(1.0)$ & $2(1.2)$ & $0(0)$ & \\
\hline Negative & $7(3.5)$ & $1(0.6)$ & $6(17.6)$ & \\
\hline Creatinine $(\mu \mathrm{mol} / 1)^{2}$ & $278(141-439)$ & $268(137-429)$ & $287(178-493)$ & 0.33 \\
\hline $\mathrm{CRP}(\mathrm{mg} / \mathrm{l})^{3}$ & $73(20-131)$ & $81(23-135)$ & $48(8-112)$ & 0.035 \\
\hline BVAS $^{4}$ & $15(12-19)$ & $15(13-19)$ & $13(10-16)$ & 0.014 \\
\hline Dialysis dependency 5 & $53(26.8)$ & $39(23.5)$ & $14(43.8)$ & 0.018 \\
\hline Renal involvement ${ }^{6}$ & $165(90.7)$ & $139(90.3)$ & $27(96.4)$ & 0.47 \\
\hline ENT involvement ${ }^{6}$ & $31(17.0)$ & $27(16.2)$ & $4(14.3)$ & 0.79 \\
\hline \multicolumn{5}{|l|}{ Country } \\
\hline Sweden & $111(55.0)$ & $103(61.7)$ & $8(22.9)$ & $<0.001$ \\
\hline UK & $53(26.2)$ & $28(16.8)$ & $25(71.4)$ & \\
\hline Czech Republic & $38(18.8)$ & $36(21.6)$ & $2(5.7)$ & \\
\hline
\end{tabular}

Demographic and clinical variables shown for all 202 patients. Comparison of demographic and clinical data between patients alive at $30 \mathrm{~d}$ with complete treatment data $(\mathrm{n}=167)$ and patients who died or did not have data on treatment $(\mathrm{n}=35)$. Values are presented as $\mathrm{n}(\%)$ or median (interquartile range) and exclude missing data. ${ }^{1}$ Data missing in 2 patients. ${ }^{2}$ Data missing in 11 patients ${ }^{3}$ Data missing in 6 patients. ${ }^{4}$ Data missing in 11 patients. ${ }^{5}$ Data missing in 4 patients. ${ }^{6}$ Data missing in 20 patients. MPA: microscopic polyangiitis; GPA: granulomatosis with polyangiitis; ANCA: antineutrophil cytoplasmic antibodies; MPO: myeloperoxidase; PR3: proteinase 3; pANCA: perinuclear ANCA; cANCA: cytoplasmic ANCA; CRP: C-reactive protein; BVAS: Birmingham Vasculitis Activity Score.

Table 2. Median dose of oral prednisolone, cyclophosphamide (CYC), and intravenous methylprednisolone the first 3 months in the different treatment groups.

\begin{tabular}{lcccc}
\hline Variables & CYC $^{1}$ & RTX $^{2}$ & No/Other & \\
\hline $\mathrm{N}(\%)$ & $112(67.1)$ & $24(14.4)$ & $31(18.6)$ & \\
Prednisolone, mg & $2570(1920-3480)$ & $1920(1300-2770)$ & $1800(670-2320)$ & $<0.001$ \\
CYC, mg & $3630(3000-6000)$ & $1000(0-2380)$ & $0(0-1040)$ & $<0.001$ \\
Methylprednisolone, mg & $0(0-750)$ & $500(0-1500)$ & $0(0-0)$ & 0.007 \\
\hline
\end{tabular}

Values are presented as median (interquartile range) unless otherwise specified. ${ }^{1}$ Cumulative CYC dose $\geq 2000$ $\mathrm{mg}$ oral CYC/ $\geq 1500 \mathrm{mg}$ intravenous CYC during the first 3 months. ${ }^{2}$ Any dose of RTX. ${ }^{3}$ No treatment, steroids only, other cytotoxic agents. RTX: rituximab.

years. The most common items were osteoporosis, diabetes, and cataract (Figure 2B). There were no cases of osteomyelitis, avascular necrosis, chemical cystitis, or gonadal failure.

In multivariable linear regression analysis, BVAS was positively associated with VDI score at 2 years, while treatment with CYC or RTX was negatively associated with VDI score (Table 3). Patients treated with CYC or RTX had a median VDI of 2 (IQR 1-3) at 2 years compared to 3 (IQR 2-4) in patients not given such treatment $(p=0.09)$. Year of diagnosis was negatively associated with VDI score in univariable, but not multivariable, analysis. The results of the analysis of VDI score at 1 year were similar to the results at 2 years.

During the first 2 years, relapses were seen in $9.6 \%$ $(14 / 146)$ of patients who reached remission after induction therapy. Relapse (as a dichotomous variable) was not associated with VDI score at 2 years in univariable analysis $(\beta 0.31,95 \% \mathrm{CI}-0.61$ to $1.23 ; \mathrm{p}=0.50)$.

Personal non-commercial use only. The Journal of Rheumatology Copyright @ 2020 . All rights reserved. 
A

Other $12 \%$

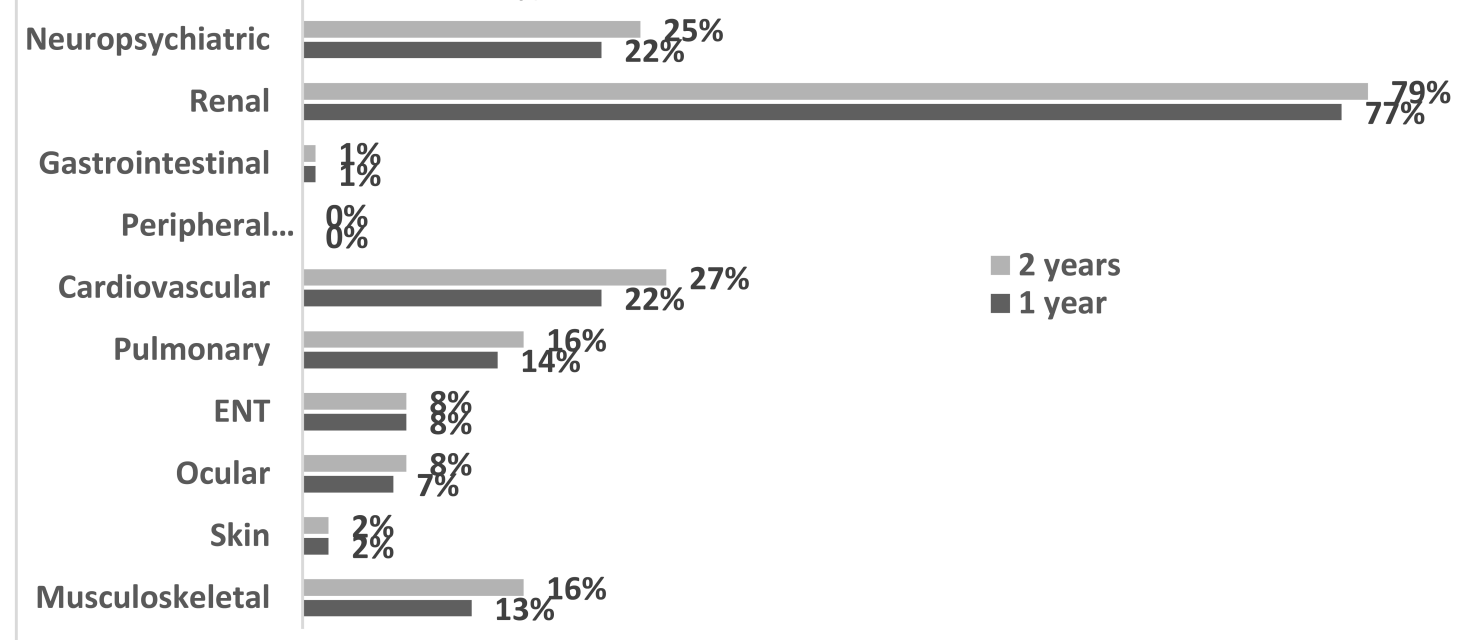

B

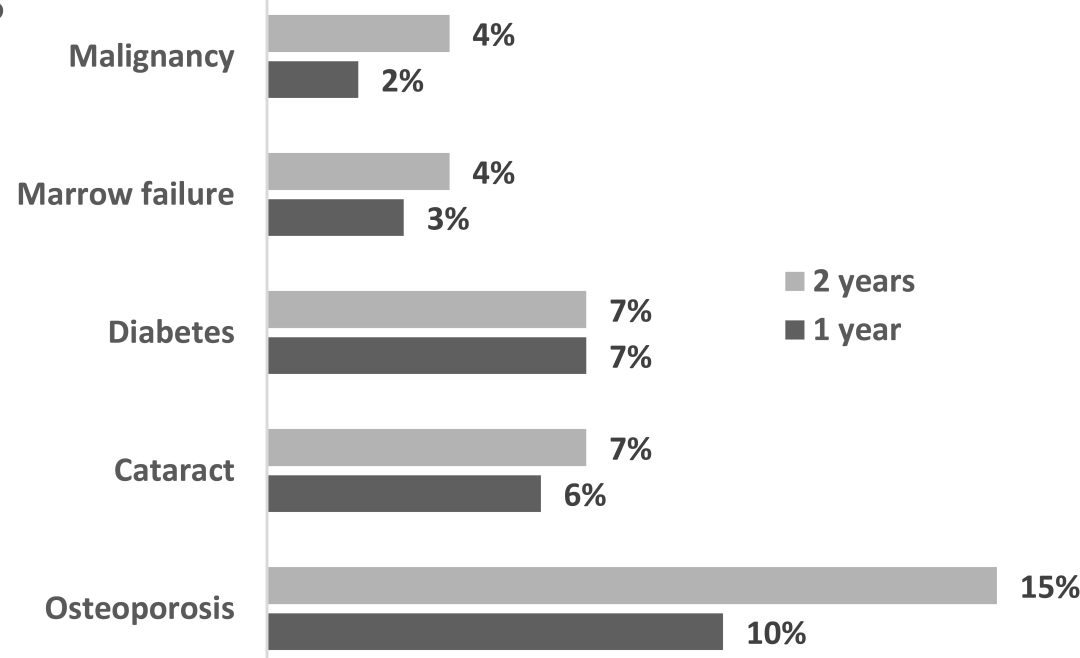

Figure 2. Vasculitis Damage Index items. A. Frequency of damage items at 1 and 2 years in patients surviving 2 years $(\mathrm{n}=108)$. "Peripheral" refers to peripheral vascular disease. B. Frequency of treatment-related damage items at 1 and 2 years in patients surviving 2 years $(n=104)$.

In multivariable binary logistic regression analysis of treatment-related items at 2 years, cumulative methylprednisolone dose was associated with increased odds of treatment-related damage (OR 1.25, 95\% CI 1.01-1.55; $\mathrm{p}=0.043$; Supplementary Table 1, available with the online version of this article). Similar results were seen at 1 year.

There were significant differences in total VDI score, but not the frequency of treatment-related damage between countries, with more damage in patients from the Czech Republic (Supplementary Table 2, available with the online version of this article).

Hospitalization. The median initial hospital period was 18 days (IQR 11-29), and during the first year 69.1\% (114/165) of the patients were readmitted to hospital, with a median stay of 12 days (IQR 6-31). Median total hospital stay (including initial stay) during the first year was 31 days (IQR 17-50). During the first year, $24.2 \%$ of the patients experienced 2 or more readmissions to the hospital. Data on causes of readmission to hospital were available for 156 of 187 readmissions in 98 of 114 patients during the first year. The most common cause was infections $(\mathrm{n}=58)$, followed by dialysis-related events $(n=18)$, cardiovascular events $(n=$ $15)$, adverse drug reactions $(\mathrm{n}=11)$, thromboembolic events $(n=9)$, relapses/active vasculitis $(n=8)$, fractures $(n=6)$, gastrointestinal bleeding/perforation $(n=4)$, diabetes $(n=4)$, and other causes $(n=23)$. 
Table 3. Univariable and multivariable linear regression analysis of Vasculitis Damage Index (VDI) score at 2 years.

\begin{tabular}{lcccc}
\hline Variables & \multicolumn{2}{c}{ Univariable Analysis } & \multicolumn{2}{c}{ Multivariable Analysis ${ }^{1}$} \\
& $\beta(95 \%$ CI $)$ & $p$ & $\beta(95 \%$ CI $)$ & $p$ \\
\hline Age (per yr) & $0.008(-0.08$ to 0.095$)$ & 0.86 & $0.026(-0.065$ to 0.12$)$ & 0.57 \\
Male sex & $-0.29(-0.85$ to 0.27$)$ & 0.31 & $-0.32(-0.89$ to 0.25$)$ & 0.26 \\
BVAS (per point) $)^{2}$ & $0.076(0.027-0.13)$ & 0.002 & $0.096(0.037-0.16)$ & 0.002 \\
MPO/pANCA & $-0.45(-1.03$ to 0.13$)$ & 0.13 & $-0.072(-0.72$ to 0.57$)$ & 0.82 \\
CRP (per percentile) & $-0.038(-0.21$ to 0.13$)$ & 0.66 & $-0.18(-0.34$ to 0.007$)$ & 0.059 \\
Creatinine (per percentile) & $0.023(-0.16$ to 0.21$)$ & 0.81 & $-0.068(-0.26$ to 0.12$)$ & 0.48 \\
Comorbidity score (per point) & $0.19(-0.17$ to 0.55$)$ & 0.29 & $0.12(-0.24$ to 0.48$)$ & 0.52 \\
CYC/RTX & $-0.82(-1.66$ to 0.022$)$ & 0.056 & $-1.16(-2.09$ to -0.23$)$ & 0.015 \\
Prednisolone dose (per percentile) & $-0.002(-0.18$ to 0.17$)$ & 0.98 & $0.005(-0.19$ to 0.20$)$ & 0.96 \\
Methylprednisolone dose (per 250 $\mathrm{mg})$ & $-0.004(-0.11$ to 0.11$)$ & 0.95 & $0.059(-0.064$ to 0.18$)$ & 0.34 \\
Year of diagnosis (per yr from 1997$)$ & $-0.09(-0.15$ to -0.025$)$ & 0.007 & $-0.046(-0.12$ to 0.025$)$ & 0.20 \\
\hline
\end{tabular}

Analysis of 108 patients with complete treatment data and data on VDI at 2 years. CYC/RTX $\geq 2000 \mathrm{mg}$ oral $\mathrm{CYC} / \geq 1500 \mathrm{mg}$ intravenous CYC or RTX. ${ }^{1}$ Multivariable analysis performed on 100 patients. ${ }^{2}$ Data missing in 2 patients. ${ }^{3}$ Data missing in 5 patients. ${ }^{4}$ Data missing in 2 patients. BVAS: Birmingham Vasculitis Activity Score; MPO: myeloperoxidase; pANCA: perinuclear antineutrophil cytoplasmic antibodies; CRP: C-reactive protein; CYC: cyclophosphamide; RTX: rituximab.

In multivariable binary logistic regression analysis of readmission, there was a significant association with MPO/pANCA positivity and creatinine level at diagnosis. MPO/pANCA positivity decreased the odds of being readmitted, while higher creatinine levels increased the odds (Table 4).

In multivariable linear regression analysis of total time spent in hospital during the first year, there was a significant association with creatinine level at diagnosis $(\beta$ 5.04, $95 \%$ CI 0.67-9.41, $\mathrm{p}=0.024$; Supplementary Table 3, available with the online version of this article).

More patients were readmitted to hospital in the Czech Republic, compared to Sweden and the UK (Supplementary Table 2, available with the online version of this article).

Cause of death. A total of 69 patients (34.2\% of 202 patients) died during the first 2 years after diagnosis. In 55 patients the cause of death was known. The most common causes of death among these were infection (34.5\%), myocardial infarction $(16.4 \%)$, and active vasculitis $(14.5 \%)$. Less common causes were gastrointestinal bleeding $(7.3 \%)$, heart failure $(7.3 \%)$, and malignancy $(7.3 \%)$. Nine patients died within 30 days from diagnosis. Of these, 5 died of vasculitis, 1 of myocardial infarction, and 1 of gastrointestinal bleeding. In 2 patients, the cause of death was unknown. Among the 19 patients who died from infection, there were no deaths within 30 days of diagnosis and 5 deaths within 90 days of diagnosis.

Analysis of death caused by infections was performed on 146 patients alive at 3 months with complete data on GC use. In multivariable Cox regression analysis, only cumulative

Table 4. Univariable and multivariable binary logistic regression analysis of rehospitalization during the first year.

\begin{tabular}{|c|c|c|c|c|}
\hline \multirow[t]{2}{*}{ Variables } & \multicolumn{2}{|c|}{ Univariable Analysis } & \multicolumn{2}{|c|}{ Multivariable Analysis 1} \\
\hline & OR $(95 \% \mathrm{CI})$ & $\mathrm{p}$ & OR $(95 \% \mathrm{CI})$ & $\mathrm{p}$ \\
\hline Age (per yr) & $0.99(0.91-1.09)$ & 0.89 & $0.99(0.89-1.10)$ & 0.82 \\
\hline Male sex & $0.73(0.37-1.41)$ & 0.34 & $0.50(0.24-1.06)$ & 0.072 \\
\hline BVAS (per point) ${ }^{2}$ & $1.05(0.98-1.11)$ & 0.16 & $1.02(0.95-1.10)$ & 0.63 \\
\hline $\mathrm{MPO} / \mathrm{pANCA}$ & $0.56(0.27-1.16)$ & 0.12 & $0.36(0.15-0.87)$ & 0.024 \\
\hline $\mathrm{CRP}(\text { per percentile })^{3}$ & $1.02(0.82-1.26)$ & 0.89 & $1.02(0.78-1.34)$ & 0.87 \\
\hline Creatinine (per percentile) $)^{4}$ & $1.22(0.98-1.52)$ & 0.072 & $1.34(1.02-1.78)$ & 0.039 \\
\hline Comorbidity score (per point) & $1.18(0.78-1.80)$ & 0.43 & $1.23(0.77-1.96)$ & 0.39 \\
\hline CYC/RTX & $1.83(0.82-4.09)$ & 0.14 & $2.21(0.82-5.94)$ & 0.12 \\
\hline Prednisolone dose (per percentile) & $1.05(0.86-1.29)$ & 0.62 & $0.99(0.76-1.30)$ & 0.96 \\
\hline Methylprednisolone dose (per $250 \mathrm{mg}$ ) & $1.07(0.93-1.23)$ & 0.37 & $1.02(0.86-1.20)$ & 0.85 \\
\hline
\end{tabular}

Analysis of 165 patients with complete treatment data and data on rehospitalization. CYC/RTX $\geq 2000 \mathrm{mg}$ oral CYC/ $\geq 1500 \mathrm{mg}$ intravenous CYC or RTX. ${ }^{1}$ Multivariable analysis performed on 148 patients. ${ }^{2}$ Data missing in 5 patients. ${ }^{3}$ Data missing in 5 patients ${ }^{4}$ Data missing in 7 patients. BVAS: Birmingham Vasculitis Activity Score; MPO: myeloperoxidase; pANCA: perinuclear antineutrophil cytoplasmic antibodies; CRP: C-reactive protein; CYC: cyclophosphamide; RTX: rituximab. 
oral PSL dose was predictive of death caused by infection (HR per percentile 1.57, 95\% CI 1.06-2.32; $\mathrm{p}=0.024$.) The results were similar in analysis of the 167 patients alive at 1 month. Median oral PSL dose in patients who died from infection was $3480 \mathrm{mg}$ (IQR 2760-4000), in patients who died from other/unknown causes $2280 \mathrm{mg}$ (IQR 1500-2730), and in patients alive after 2 years $2290 \mathrm{mg}$ (IQR 1800-3190, $\mathrm{p}=0.01$ ).

There was no significant difference between countries regarding the frequency of deaths caused by infections (Supplementary Table 2, available with the online version of this article).

\section{DISCUSSION}

The aim of our study was to investigate associations between permanent organ damage, hospital admissions, causes of death, and the treatment given during the first months after diagnosis in elderly patients with MPA and GPA. The most important finding is that elderly patients treated with adequate doses of CYC or RTX developed less permanent organ damage compared to patients who were treated with less aggressive regimens and patients who received no treatment. The most probable cause for this is that the immunosuppressive treatment halts the inflammatory process and that damage accrued over time is mainly caused by the disease and not the treatment. There was no association between treatment and rehospitalization or total hospital stay during the first year after diagnosis.

In a previous study by our group, largely based on the same cohort, we found that the patients who had received treatment with RTX or CYC had better survival at 2 years ${ }^{13}$. The concern that this higher survival would be at the expense of increased morbidity and healthcare use was thus not confirmed in our current study.

A selected number of VDI items are considered treatment-related ${ }^{18}$, and these were seen in about one-fourth of the patients. When limiting the analysis to these items, there was no association between damage and treatment with CYC or RTX, or the total oral PSL dose. However, there was a positive association between treatment-related damage and cumulative methylprednisolone dose. Previous studies in younger patients have shown an association between organ damage and higher cumulative doses and longer duration of GC use ${ }^{10,12}$. A recently published retrospective study of patients with severe AAV with renal involvement found an association between treatment with intravenous methylprednisolone and higher incidence of diabetes mellitus ${ }^{20}$, one of the damage items considered treatment-related.

Another important finding in our study is that the patients who died from infections had received higher doses of oral GC compared to patients who died from other causes or who survived the first 2 years, and this was the only significant factor associated with death caused by infections in multivariable analysis. Infections were the most common cause of death in this elderly population, as previously shown in younger patients ${ }^{7}$, and in smaller studies of elderly patients $^{21,22}$. Patients with AAV have high rates of severe infections compared to the general population, and the risk increases with advancing age ${ }^{23}$. Most of the deaths caused by infections occurred after the first 3 months of treatment. Because we did not have data on GC use beyond 3 months, we are not able to discern whether this observed association was due to a continued trend of longer or higher cumulative dose of GC during the first year. Further studies are needed before any firm conclusions can be made on whether oral or IV GC are associated with the greatest risks in elderly patients. However, our results do raise concerns regarding the use of high doses of $\mathrm{GC}$ regarding treatment-related damage and severe infections. A regimen with lower CYC dose and faster tapering of GC was associated with lower risk for serious adverse events, while deaths, remission, and relapse rates did not differ significantly, in a cohort of patients with AAV aged 65 years or more ${ }^{24}$.

We found high readmission numbers among the patients included in this study; $69 \%$ were readmitted to the hospital during the first year after diagnosis. This can be compared to readmission rates of $71 \%$ in advanced cancer patients ${ }^{25}$ and $58 \%$ in older patients on chronic hemodialysis ${ }^{26}$. There are few studies of hospitalization patterns in AAV. Wallace, et $a l^{27}$ studied patients in all age groups and found that the median length of stay in hospital with a primary diagnosis of GPA was 6.2 days. This can be compared to the total median hospital stay of 31 days and the median stay during readmission of 12 days found in the present study. In the general population in Sweden aged 75 years or more, the mean hospital stay during 2013 was 6.3 days $^{14}$. In a Danish population-based study, the risk of infection-related hospitalization was 9.5 times higher among patients with GPA compared to population controls during the first year of followup ${ }^{28}$. In line with those results, infections were most common among known causes of rehospitalization in our cohort.

In multivariable analysis, $\mathrm{MPO} / \mathrm{pANCA}$ positivity decreased the odds of readmission to hospital during the first year, while poorer renal function at diagnosis was associated with increased odds of readmission. The negative association between MPO/pANCA positivity and readmission was not seen in the univariable analysis, but in the multivariable analysis after adjusting for creatinine. The most probable reason for this finding is that the MPO/pANCA-positive patients had less extrarenal involvement causing readmission. Creatinine level at diagnosis was also associated with total time spent in hospital during the first year, showing the importance and severity of renal impairment in these elderly patients.

A great majority of the patients had some type of permanent damage at 2 years of followup, although multiple types of damage were seen in only a minority of the patients.

Personal non-commercial use only. The Journal of Rheumatology Copyright $\subset$ 2020. All rights reserved. 
Higher BVAS at diagnosis was associated with higher VDI score at followup, reflecting the effect of disease activity on subsequent damage. This is in line with the results from the European Vasculitis Study Group trials ${ }^{10}$.

We found damage to be most common in the renal and cardiovascular domains, a finding similar to previously published studies ${ }^{9,11}$. However, ENT damage was seen less frequently, because GPA with ENT involvement was less common in our cohort. This is expected given the older age of the patients in our study. MPA is more common than GPA in older patients ${ }^{6,22,29,30}$, and older patients have more renal and less ENT involvement ${ }^{21,31}$. Of the centers participating in the present study, a majority mainly recruits nephrology patients. However, referral bias is not the only explanation for the predominance of renal involvement because it did not differ between countries, and was high also in the countries recruiting from rheumatology units ${ }^{13}$.

The main limitation of our study is that data are retrospective and that the patients have not been randomized to treatment. Owing to the retrospective collection of data, information on treatment, causes of death, and causes of rehospitalization was not complete for all patients included. There is a risk of confounding by indication in the treatment data, because the motive for treatment decisions is not known. When analyzing and interpreting the data, it was presumed that the therapy given during the first 30 days was intended to continue in accordance with recommendations on immunosuppressive treatment ${ }^{32,33}$. However, we cannot exclude the possibility that patients who were perceived as having a poorer prognosis because of comorbidities or frailty were not treated according to recommendations and that these patients also developed more permanent damage. Neither can we rule out the possibility that patients who were perceived as having an aggressive disease received more intensive treatment, including higher doses of GC. These severely ill patients were likely to be at higher risk for adverse events and damage.

The risk of survival bias also needs to be acknowledged. Patients with more damage have an increased mortality risk ${ }^{18}$ and patients with severe damage resulting in early death are thus not included in the analyses of VDI. However, the starting point for this study was to investigate damage in surviving patients. Our previous study in elderly patients showed reduced mortality in treated patients, making survival bias an unlikely cause for the negative association between treatment and damage.

We found that a majority of elderly patients with MPA and GPA developed permanent organ damage over time and that the frequency of readmission during the first year after diagnosis was high. Treatment with CYC and RTX was associated with development of less permanent organ damage and was not associated with readmission to hospital or total time spent in hospital during the first year after diagnosis. However, IV GC were associated with treatment-related damage and oral corticosteroids given during the first 3 months with increased risk of fatal infections.

\section{ACKNOWLEDGMENT}

The authors thank Qianwen Ko (Royal Free Hospital, London) and Doubravka Frausova (Charles University and General University Hospital, Prague) for their acquisition of data.

\section{ONLINE SUPPLEMENT}

Supplementary material accompanies the online version of this article.

\section{REFERENCES}

1. Jennette JC, Falk RJ, Hu P, Xiao H. Pathogenesis of antineutrophil cytoplasmic autoantibody-associated small-vessel vasculitis. Annu Rev Pathol 2013;8:139-60.

2. Booth AD, Almond MK, Burns A, Ellis P, Gaskin G, Neild GH, et al; Pan-Thames Renal Research Group. Outcome of ANCA-associated renal vasculitis: a 5-year retrospective study. Am J Kidney Dis 2003;41:776-84.

3. Mohammad AJ, Jacobsson LT, Westman KW, Sturfelt G, Segelmark $M$. Incidence and survival rates in Wegener's granulomatosis, microscopic polyangiitis, Churg-Strauss syndrome and polyarteritis nodosa. Rheumatology 2009;48:1560-5.

4. Ntatsaki E, Watts RA, Scott DG. Epidemiology of ANCA-associated vasculitis. Rheum Dis Clin North Am 2010;36:447-61.

5. Heijl C, Harper L, Flossmann O, Stucker I, Scott DG, Watts RA, et al; European Vasculitis Study Group (EUVAS). Incidence of malignancy in patients treated for antineutrophil cytoplasm antibody-associated vasculitis: Follow-up data from European Vasculitis Study Group clinical trials. Ann Rheum Dis 2011;70:1415-21.

6. Flossmann O, Berden A, de Groot K, Hagen C, Harper L, Heijl C, et al; European Vasculitis Study Group. Long-term patient survival in ANCA-associated vasculitis. Ann Rheum Dis 2011;70:488-94.

7. Little MA, Nightingale P, Verburgh CA, Hauser T, De Groot K, Savage C, et al; European Vasculitis Study (EUVAS) Group. Early mortality in systemic vasculitis: relative contribution of adverse events and active vasculitis. Ann Rheum Dis 2010;69:1036-43.

8. Exley AR, Bacon PA, Luqmani RA, Kitas GD, Gordon C, Savage $\mathrm{CO}$, et al. Development and initial validation of the Vasculitis Damage Index for the standardized clinical assessment of damage in the systemic vasculitides. Arthritis Rheum 1997;40:371-80.

9. Robson J, Doll H, Suppiah R, Flossmann O, Harper L, Höglund P, et al. Damage in the ANCA-associated vasculitides: long-term data from the European Vasculitis Study Group (EUVAS) therapeutic trials. Ann Rheum Dis 2015;74:177-84.

10. Robson J, Doll H, Suppiah R, Flossmann O, Harper L, Hoglund P, et al. Glucocorticoid treatment and damage in the anti-neutrophil cytoplasm antibody-associated vasculitides: long-term data from the European Vasculitis Study Group trials. Rheumatology 2015;54:471-81.

11. Mohammad AJ, Bakoush O, Sturfelt G, Segelmark M. The extent and pattern of organ damage in small vessel vasculitis measured by the Vasculitis Damage Index (VDI). Scand J Rheumatol 2009;38:268-75.

12. Koldingsnes $\mathrm{W}$, Nossent $\mathrm{H}$. Predictors of survival and organ damage in Wegener's granulomatosis. Rheumatology 2002;41:572-81.

13. Weiner M, Goh SM, Mohammad AJ, Hruskova Z, Tanna A, Bruchfeld A, et al. Outcome and treatment of elderly patients with ANCA-associated vasculitis. Clin J Am Soc Nephrol 2015; 10:1128-35.

14. Socialstyrelsen. [Statistics database for diagnoses in closed care]. [Article in Swedish] [Internet. Accessed October 30, 2019.]

Personal non-commercial use only. The Journal of Rheumatology Copyright (c) 2020. All rights reserved. 
Available from: sdb.socialstyrelsen.se/if_par/val.aspx

15. Watts R, Lane S, Hanslik T, Hauser T, Hellmich B, Koldingsnes W, et al. Development and validation of a consensus methodology for the classification of the ANCA-associated vasculitides and polyarteritis nodosa for epidemiological studies. Ann Rheum Dis 2007;66:222-7.

16. Flossmann O, Bacon P, de Groot K, Jayne D, Rasmussen N, Seo P, et al. Development of comprehensive disease assessment in systemic vasculitis. Ann Rheum Dis 2007;66:283-92.

17. Levey AS, Bosch JP, Lewis JB, Greene T, Rogers N, Roth D. A more accurate method to estimate glomerular filtration rate from serum creatinine: a new prediction equation. Modification of Diet in Renal Disease Study Group. Ann Intern Med 1999;130:461-70.

18. Exley AR, Bacon PA, Luqmani RA, Kitas GD, Carruthers DM, Moots R. Examination of disease severity in systemic vasculitis from the novel perspective of damage using the Vasculitis Damage Index (VDI). Br J Rheumatol 1998;37:57-63.

19. Davies SJ, Russell L, Bryan J, Phillips L, Russell GI. Comorbidity, urea kinetics, and appetite in continuous ambulatory peritoneal dialysis patients: their interrelationship and prediction of survival. Am J Kidney Dis 1995;26:353-61.

20. Chanouzas D, McGregor JA, Nightingale P, Salama AD, Szpirt WM, Basu N, et al. Intravenous pulse methylprednisolone for induction of remission in severe ANCA associated vasculitis: a multi-center retrospective cohort study. BMC Nephrol 2019;20:58

21. Sato S, Yashiro M, Matsuoka N, Asano T, Kobayashi H, Watanabe $\mathrm{H}$, et al. Clinical features and outcomes in patients with elderly-onset anti-neutrophil cytoplasmic antibody-associated vasculitis. Geriatr Gerontol Int 2018;18:1453-7.

22. Hoganson DD, From AM, Michet CJ. ANCA vasculitis in the elderly. J Clin Rheumatol 2008;14:78-81.

23. Mohammad AJ, Segelmark M, Smith R, Englund M, Nilsson JA Westman K, et al. Severe infection in antineutrophil cytoplasmic antibody-associated vasculitis. J Rheumatol 2017;44:1468-75.

24. Pagnoux C, Quemeneur T, Ninet J, Diot E, Kyndt X, de Wazieres B, et al; French Vasculitis Study Group. Treatment of systemic necrotizing vasculitides in patients aged sixty-five years or older: results of a multicenter, open-label, randomized controlled trial of corticosteroid and cyclophosphamide-based induction therapy. Arthritis Rheumatol 2015;67:1117-27.

25. Whitney RL, Bell JF, Tancredi DJ, Romano PS, Bold RJ, Joseph JG. Hospitalization rates and predictors of rehospitalization among individuals with advanced cancer in the year after diagnosis. J Clin Oncol 2017;35:3610-7.

26. Schoonover KL, Hickson LJ, Norby SM, Hogan MC, Chaudhary S, Albright RC Jr, et al. Risk factors for hospitalization among older, incident haemodialysis patients. Nephrology 2013;18:712-7.

27. Wallace ZS, Lu N, Miloslavsky E, Unizony S, Stone JH, Choi HK Nationwide trends in hospitalizations and in-hospital mortality in granulomatosis with polyangiitis (Wegener's). Arthritis Care Res 2017;69:915-21.

28. Faurschou M, Obel N, Baslund B. Long-term risk and outcome of infection-related hospitalization in granulomatosis with polyangiitis: a nationwide population-based cohort study. Scand J Rheumatol 2018;47:475-80

29. Harper L, Savage CO. ANCA-associated renal vasculitis at the end of the twentieth century - a disease of older patients. Rheumatology 2005;44:495-501.

30. Chen M, Yu F, Zhang Y, Zhao MH. Antineutrophil cytoplasmic autoantibody-associated vasculitis in older patients. Medicine 2008;87:203-9.

31. Krafcik SS, Covin RB, Lynch JP 3rd, Sitrin RG. Wegener's granulomatosis in the elderly. Chest 1996;109:430-7.

32. Mukhtyar C, Guillevin L, Cid MC, Dasgupta B, de Groot K, Gross W, et al; European Vasculitis Study Group. EULAR recommendations for the management of primary small and medium vessel vasculitis. Ann Rheum Dis 2009;68:310-7.

33. Stone JH, Merkel PA, Spiera R, Seo P, Langford CA, Hoffman GS, et al. Rituximab versus cyclophosphamide for ANCA-associated vasculitis. N Engl J Med 2010;363:221-32. 